\title{
Motional narrowing effect in one-dimensional Frenkel chains with configurational disorder
}

\author{
V. A. Malyshev ${ }^{\dagger}$ and F. Domínguez-Adame \\ GISC, Departamento de Física de Materiales, Universidad Complutense, E-28040 Madrid, Spain
}

(February 1, 2008)

\begin{abstract}
We analyze the peculiarities of the motional narrowing effect in disordered one-dimensional Frenkel chains with off-diagonal disorder, induced by uncorrelated Gaussian fluctuations in the positions of the host units. A clear difference in the scaling laws with respect to the magnitude of the positional disorder and the chain size is found, as compared to those for uncorrelated diagonal disorder as well as for off-diagonal disorder modeled by uncorrelated randomness in the nearest-neighbor couplings. The origin of such a difference is discussed in detail.
\end{abstract}

PACS number(s): 71.35.Aa; 36.20.Kd; 78.30.Ly 


\section{INTRODUCTION}

The concept of motional narrowing, earlier raised by Knapp [1] for the on-site energy (or diagonal) disorder, has been very fruitful in explaining many optical phenomena in quasione-dimensional systems like $\mathrm{J}$ aggregates of polymethine dyes and conjugated polymers

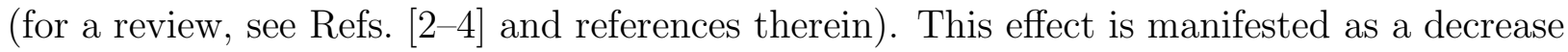
of the magnitude of the diagonal disorder as soon as the states of individual molecules are collectivized due to the intermolecular interaction and form excitonic states. The decrease depends on whether or not the disorder is small enough to be regarded as a perturbation. In the perturbative case, the suppression factor is determined by the square root of the whole number of molecules in an aggregate, while in the nonperturbative case this number should be substituted by the number of coherently bound molecules [1]. A similar narrowing effect, but different suppression factor, was recently reported for the case of dynamic disorder [5 [7].

It was found in Ref. [8] that the numerically simulated absorption spectrum of polysilane with an uncorrelated Gaussian distribution of nearest-neighbor couplings are similar to those for an uncorrelated diagonal disorder (see also [9]). In contrast, numerical simulations of off-diagonal disorder given by Gaussian randomness in the molecular positions [2,10] found that the different behavior of the optical observables was very different from that expected from standard motional narrowing arguments [1].

The main goal of the present Letter is to uncover the origin of such a difference. It will be shown that, in the case of configurational disorder, certain correlations appear in the distribution of hopping integrals, in spite of the fact that the distribution of the molecular positions is uncorrelated. This finally results in a scaling law for the typical fluctuation of the Frenkel Hamiltonian matrix with respect to the magnitude of positional disorder and the number of molecules different from that for uncorrelated diagonal disorder. As a consequence, the main features of the exciton optical response are largely affected. 


\section{DESCRIPTION OF THE MODEL}

Consider a collection of $N$ two-level molecules forming a one-dimensional lattice. For our present purposes, we will neglect the static inhomogeneous offset energy of the molecules imposed by the surrounding host medium (diagonal disorder). Under this assumption, the effective Frenkel Hamiltonian describing the system can be written in the form

$$
\mathcal{H}=\sum_{n m} J_{n m}|n\rangle\langle m|
$$

Here, the state vector $|n\rangle$ denotes the $n$-th molecule being excited and the site index $n$ lies within the symmetric domain $-(N-1) / 2 \leq n \leq(N-1) / 2(N$ assumed to be odd). $J_{n m}$ is the intersite interaction, which will be considered to be of dipole origin, so that $J_{n m}=J /\left|n-m+\xi_{n}-\xi_{m}\right|^{3}, n \neq m$, and $J_{n n} \equiv 0 ; \xi_{n}$ is the deviation of the $n$-th molecule from its regular position, which we suppose that it occurs only along the directions toward the two adjacent molecules. This restriction allows us to replace the fluctuations of vector positions by scalars. We note that, arguably, this assumption does not affect the conclusions and is introduced only to simplify the analytical treatment. The distribution function of $\xi_{n}$ is chosen to be Gaussian,

$$
P\left(\xi_{n}\right)=\frac{1}{\sigma \sqrt{2 \pi}} \exp \left(-\frac{\xi_{n}^{2}}{2 \sigma^{2}}\right)
$$

with variance $\sigma^{2}$.

To study the motional narrowing effect, one should rewrite the Hamiltonian (1) in the excitonic representation using the eigenfunctions of the unperturbed Hamiltonian (with no

disorder). For the sake of simplicity, we assume periodic boundary conditions. Then, Bloch plane waves are the proper eigenfunctions of (1) in the absence of disorder:

$$
|K\rangle=\frac{1}{\sqrt{N}} \sum_{n} e^{i K n}|n\rangle,
$$

where $K=2 \pi k / N$ belongs to the first Brillouin zone $(-(N-1) / 2 \leq k \leq(N-1) / 2)$.

In the $K$-representation, the Hamiltonian (11) reads 


$$
\begin{aligned}
\mathcal{H} & =\sum_{K} E_{K}|K\rangle\left\langle K\left|+\sum_{K, K^{\prime}} \Delta_{K K^{\prime}}\right| K\right\rangle\left\langle K^{\prime}\right|, \\
E_{K} & =2 J \sum_{n>0} \frac{1}{n^{3}} \cos (K n), \\
\Delta_{K K^{\prime}} & =\frac{1}{N} \sum_{m n} \delta J_{n m} e^{i\left(K n-K^{\prime} m\right)}, \\
\delta J_{n m} & =J\left(\frac{1}{\left|n-m+\xi_{n}-\xi_{m}\right|^{3}}-\frac{1}{|n-m|^{3}}\right),
\end{aligned}
$$

where the scattering matrix $\Delta_{K K^{\prime}}$ is real due to the symmetry of the summation region in (4c). Its diagonal part gives rise to exciton energy shift, yielding inhomogeneous broadening of exciton levels for an ensemble of chains. This effect appears to be the main consequence of the presence of disorder if the off-diagonal term can be regarded as a perturbation. The typical fluctuation of $\Delta_{K K}$ has a direct relation with the inhomogeneous width of the corresponding exciton state $|K\rangle$.

For nonperturbative magnitudes of the disorder, the off-diagonal elements $\Delta_{K K^{\prime}}(K \neq$ $K^{\prime}$ ) mix the exciton states, resulting in their localization on chain segments of a typical size smaller than the chain length and subsequently affecting the exciton optical response. Recall that, for a perfect chain, only the state with $K=0$ is coupled to the light and carries the entire exciton oscillator strength, which is then $N$ times larger than that for an isolated molecule. Being mixed with other (nonradiative) states $(K \neq 0)$, the radiative state loses a part of the oscillator strength due to its spreading over to the nonradiative ones. Thus, an effective number, usually called number of coherently bound molecules $N^{*}<N$, replaces the system size as the enhancement factor of the oscillator strength of the localized exciton states [1]. It reflects the typical number of sites on which the localized exciton wave functions have a significant magnitude or, in other words, the number of molecules within a typical localization segment. Accordingly, the inhomogeneous width of the optical exciton line will also be subjected to renormalization [1]. In the next Section, we will formulate a condition separating the perturbative and nonperturbative ranges of disorder magnitudes in order to treat the exciton optical response. 


\section{MOTIONAL NARROWING EFFECT}

To gain insight into the magnitude of the typical fluctuation of the scattering matrix $\Delta_{K K^{\prime}}$, one should calculate either its distribution function or its moments, using the distribution (2) of the positional fluctuations. We chose the second procedure, so that the magnitude of interest will be the mean square deviation, defined as

$$
\sigma_{K K^{\prime}}^{2}=\left\langle\Delta_{K K^{\prime}}^{2}\right\rangle-\left\langle\Delta_{K K^{\prime}}\right\rangle^{2}
$$

where brackets denote the average over the joint probability distribution $\prod_{n} P\left(\xi_{n}\right)$, with $P\left(\xi_{n}\right)$ of the form (2).

In what follows, we assume that the standard deviation $\sigma$ is small. Accounting then for that

$$
\frac{1}{\left|n-m+\xi_{n}-\xi_{m}\right|^{3}}=\frac{1}{|n-m|^{3}}\left[1+\frac{2(n-m)\left(\xi_{n}-\xi_{m}\right)}{(n-m)^{2}}+\frac{\left(\xi_{n}-\xi_{m}\right)^{2}}{(n-m)^{2}}\right]^{-3 / 2}
$$

one can expand $\delta J_{n m}$ in Eq. (4di) in Taylor series up to fourth order with respect to

$$
X_{n m} \equiv \frac{2(n-m)\left(\xi_{n}-\xi_{m}\right)+\left(\xi_{n}-\xi_{m}\right)^{2}}{(n-m)^{2}} ;
$$

we are interested in the contribution to $\sigma_{K K^{\prime}}^{2}$ up to the same order. The corresponding expressions for $\Delta_{K K^{\prime}}$ and $\Delta_{K K^{\prime}}^{2}$ read

$$
\begin{aligned}
\Delta_{K K^{\prime}} & =\frac{J}{N} \sum_{m n} \frac{e^{i\left(K n-K^{\prime} m\right)}}{|n-m|^{3}}\left(-\frac{3}{2} X_{n m}+\frac{15}{8} X_{n m}^{2}-\frac{35}{16} X_{n m}^{3}+\frac{315}{128} X_{n m}^{4}\right), \\
\Delta_{K K^{\prime}}^{2} & =\left(\frac{J}{N}\right)^{2} \sum_{m n} \frac{e^{i\left(K n-K^{\prime} m\right)}}{|n-m|^{3}} \sum_{p q} \frac{\left.e^{-i\left(K q-K^{\prime} p\right.}\right)}{|q-p|^{3}} \\
& \times\left(\frac{9}{4} X_{n m} X_{q p}-\frac{45}{8} X_{n m} X_{q p}^{2}+\frac{105}{16} X_{n m} X_{q p}^{3}+\frac{225}{64} X_{n m}^{2} X_{q p}^{2}\right) .
\end{aligned}
$$

Carrying out the average in Eqs. (6), we will collect all terms up to fourth order in $\sigma$. The calculations are rather tedious but straightforward, so we only quote the final results: 


$$
\begin{aligned}
\left\langle\Delta_{K K^{\prime}}\right\rangle & =J \delta_{K K^{\prime}}\left[12 J Q_{5}(K) \sigma^{2}+180 Q_{7}(K) \sigma^{4}\right], \\
\left\langle\Delta_{K K^{\prime}}^{2}\right\rangle= & \frac{9 J^{2} \sigma^{2}}{N}\left|P_{5}(K)+P_{5}^{*}\left(K^{\prime}\right)\right|^{2}+144 J^{2} \sigma^{4} Q_{5}^{2}(K) \delta_{K K^{\prime}} \\
& +\frac{360 J^{2} \sigma^{4}}{N}\left[P_{5}(K)+P_{5}^{*}\left(K^{\prime}\right)\right]\left[P_{7}^{*}(K)+P_{7}\left(K^{\prime}\right)\right] \\
& +\frac{72 J^{2} \sigma^{4}}{N}\left[\left[Q_{5}(K)+Q_{5}\left(K^{\prime}\right)\right]^{2}+2\left[Q_{10}(0)+Q_{10}\left(K+K^{\prime}\right)\right]\right],
\end{aligned}
$$

where the functions $Q_{\nu}(K)$ and $P_{\nu}(K)$ are given by

$$
P_{\nu}(K)=\sum_{m} \frac{m e^{i K m}}{|m|^{\nu}}, \quad Q_{\nu}(K)=\sum_{m} \frac{e^{i K m}}{|m|^{\nu}},
$$

and the term $m=0$ is excluded in the summations.

For the magnitude of interest, one obtains

$$
\begin{aligned}
\sigma_{K K^{\prime}}^{2} & =\frac{9 J^{2} \sigma^{2}}{N}\left|P_{5}(K)+P_{5}^{*}\left(K^{\prime}\right)\right|^{2}+\frac{360 J^{2} \sigma^{4}}{N}\left[P_{5}(K)+P_{5}^{*}\left(K^{\prime}\right)\right]\left[P_{7}^{*}(K)+P_{7}\left(K^{\prime}\right)\right] \\
& +\frac{72 J^{2} \sigma^{4}}{N}\left[\left[Q_{5}(K)+Q_{5}\left(K^{\prime}\right)\right]^{2}+2\left[Q_{10}(0)+Q_{10}\left(K+K^{\prime}\right)\right]\right] .
\end{aligned}
$$

We stress that only terms up to fourth order in $\sigma$ are kept.

To treat the optical properties of linear aggregates, the important wavenumbers $K$ and $K^{\prime}$ are the smaller ones, namely $|K|,|K|^{\prime} \ll 1$. In this long wavelength limit, the functions $P_{\nu}(K)$ and $Q_{\nu}(K)$ can be approximated by their values within the nearest-neighbor framework, namely $P_{\nu}(K) \approx 2 i K$ and $Q_{\nu}(K) \approx 2$, accounting in the sums (8) only for leading terms with $m= \pm 1$. Equation (9) then reduces to

$$
\sigma_{K K^{\prime}}^{2}=\frac{36 J^{2}}{N}\left(K-K^{\prime}\right)^{2} \sigma^{2}\left(1+40 \sigma^{2}\right)+\frac{1728 J^{2}}{N} \sigma^{4}
$$

Now it can be clearly seen why one should keep the terms up to fourth order in $\sigma$. The first term, being of second order with respect to $\sigma$, is equal to zero for the diagonal elements of 
$\sigma_{K K^{\prime}}$ [note that it is true independently of the magnitude of $K$ since $P_{\nu}(K)+P_{\nu}^{*}(K) \equiv 0$ ]. Thus, for $\sigma_{K K}^{2}$ one gets

$$
\sigma_{K K}^{2}=\frac{1728 J^{2}}{N} \sigma^{4}
$$

This result differs from that for uncorrelated diagonal disorder, where the corresponding magnitude scales as $\sigma^{2} / N, \sigma^{2}$ being the variance of the site energy distribution [1]. The same behavior appears as well when one simulates off-diagonal disorder by uncorrelated randomness in the nearest-neighbor hopping integrals [8.9] (here, $\sigma^{2}$ stands for the variance of the corresponding distribution).

With respect to the off-diagonal elements of $\sigma_{K K^{\prime}}^{2}$, we should note that, in spite of the fact that the first term scales as $\sigma^{2}$, it has an additional suppression factor proportional to $\left(K-K^{\prime}\right)^{2} \sim N^{-2}$, and thus it may be smaller than the fourth order one. When the first term in Eq. (10) dominates $\sigma_{K K^{\prime}}^{2} \propto \sigma^{2} / N^{3}$, while in the opposite case one has $\sigma_{K K^{\prime}}^{2} \propto \sigma^{4} / N$. Both results also differ from the scaling law $\sigma^{2} / N$ found for the other types of disorder [1,80.97.

The origin of the difference discovered lies in that the terms linear in $\xi_{n}$ in the fluctuations of the dipolar coupling of site $n$ to the adjacent ones have the same magnitude but opposite signs, thus appearing to be correlated, notwithstanding the fact that the fluctuations of molecular positions are completely uncorrelated. Due to this feature, they almost (or exactly at $K=K^{\prime}$ ) cancel each other in (4a) when summing over $n$ in the long wavelength limit $\left(|K|,\left|K^{\prime}\right| \ll 1\right)$.

As it was noted in the previous Section, for perturbative magnitudes of disorder, the main effect of $\sigma_{K K^{\prime}}$ is the broadening of the exciton levels. The value of $\sigma_{K K}$ given by Eq. (11) gives the half width of the $K$-th exciton state and will serve for determining the latter provided that $\sigma_{K K^{\prime}}$ remains smaller than the corresponding energy differences $\left|E_{K}-E_{K^{\prime}}\right|$. Since the minimum energy difference in the exciton spectrum is between the state with $K=0$ and the next one with $K=2 \pi / N$, the equality

$$
\sigma_{K=2 \pi / N, K^{\prime}=0}=\left|E_{K=2 \pi / N}-E_{K^{\prime}=0}\right|
$$


determines a value of $\sigma$ which separates the ranges of perturbative and nonperturbative magnitudes of disorder. In (12), $E_{K}$ is given by Eq. (4b).

\section{DISCUSSION OF THE NUMERICAL RESULTS}

As it was already mentioned in the Introduction, numerical simulations of optical properties of linear molecular aggregates with off-diagonal disorder generated by Gaussian uncorrelated fluctuations in the molecular positions yielded different behaviors of the optical observables as compared to those for diagonal disorder [2,10]. In this Section, it will be shown that the peculiarities found in Refs. [2,10] can be qualitatively explained from the viewpoint of the modified motional narrowing formula (10). In particular, we will focus on the dependence of the absorption band width $\sigma^{*}$ and the radiative rate enhancement factor on the degree of disorder $\sigma$. The radiative rate enhancement factor is proportional to the number of coherently bound molecules $N^{*}$, while $\sigma^{*}$ can be estimated from $\sigma_{K K}$ replacing $N$ by $N^{*}$ [1:[1]. Thus, the magic number $N^{*}$ is, in fact, the unique quantity determining the observables we are interested in and, correspondingly, our main goal is to obtain the dependence of $N^{*}$ on $\sigma$. To do that, we will follow a simple rule earlier proposed in Ref. 12 (see also [11]) that works surprisingly well for explaining the corresponding data for diagonal disorder [11,12]. This rule simply consists of applying the formula (12) to the typical localization segment of size $N^{*}$. In our estimates of $N^{*}$, we will keep only the second term in Eq. (10) since, as will be shown later, it is the major contribution for the parameters used in Refs. [2, 10].

As a first step, let us take the exciton energy spectrum in the nearest-neighbor approximation: $E_{K}=2 J \cos K \approx 2 J-J K^{2}$. After these simplifications, we arrive at the following formula for the number of coherently bound molecules

$$
N^{*}=\left(\frac{\pi^{4}}{108}\right)^{1 / 3} \sigma^{-4 / 3} \approx \sigma^{-4 / 3}
$$

Estimating now the absorption band width $\sigma^{*}$ as $2 \sigma_{K K}$ by replacing $N$ by $N^{*}$, we find 


$$
\sigma^{*}=2 \sqrt{1728} J \sigma^{8 / 3} \approx 83 J \sigma^{8 / 3}
$$

Recall that for diagonal disorder the respective quantities scale as $\sigma^{-2 / 3}$ [11, 12 and $\sigma^{4 / 3}[2,10$ [15], whenever the nearest-neighbor approximation is adopted. It is worth noting that the new scaling laws (13) and (14) follow from the latters $\left(\sigma^{-2 / 3}\right.$ and $\left.\sigma^{4 / 3}\right)$ simply replacing $\sigma$ by $\sigma^{2}$, reflecting the fact that in our case the effective disorder scales as $\sigma^{2}$ instead of $\sigma$ [see Eq. (11)].

The authors of Refs. [2,10] did not restrict themselves to the nearest-neighbor approximation but took into account all dipolar couplings. Using the parameterization $c \sigma^{\alpha}$, they found that their numerical data for the factor of radiative rate enhancement and $\sigma^{*}$ were fitted by the sets $c=0.20, \alpha=-1.64$ and $c=425 J, \alpha=2.84$, respectively. The corresponding exponents for the case of diagonal disorder were found to be -0.74 and 1.34 , respectively. Here, one also observes approximately the two-times increase of the exponents when passing from diagonal to off-diagonal disorder. This feature unambiguously shows that the effective degree of disorder in the last case scales as $\sigma^{2}$, what perfectly correlates with our finding given by Eq. (11).

Comparison of the numerical fits of Refs. [2, [10] with our results (13) and (14), obtained under the assumption of nearest-neighbor coupling, shows that the numerical $\sigma$-scaling of both quantities is reproduced reasonably well by the theoretical estimates, better for the absorption band width and worse for the number of coherently bound molecules. Similar peculiarities are present in the case of diagonal disorder (compare 4/3 with 1.34 and $-2 / 3$ with -0.74). As it was firstly mentioned in Refs. [2, 10, including all dipolar couplings affects largely the factor of radiative rate enhancement, rising it by more than a factor 2 as compared to that calculated in the nearest-neighbor approximation. The explanation was done in Ref. [16] and was based on the exact exciton energy spectrum close to the bottom of the band

$$
E_{K}=-2 J \xi(3)+J K^{2}\left(\frac{3}{2}-\ln K\right)
$$

where $\xi(3)=\sum_{n=1}^{\infty} n^{-3}=1.202$. Notice that the presence of a logarithmic term in this 
equation results in a larger energy level separation as compared to the case of the nearestneighbor model. Thus, a smaller number of the exciton states will be effectively mixed by disorder giving finally rise to an increase of $N^{*}$. Indeed, if we now use Eq. (15) for finding $N^{*}$ we then arrive at the equation

$$
\frac{N^{*} \sqrt{N^{*}}}{\ln N^{*}-\ln (2 \pi)+3 / 2}=\frac{\pi^{2}}{6 \sqrt{3} \sigma^{2}} .
$$

For the nearest-neighbor model, one should substitute the denominator in the left hand side of Eq. (16) by unity, getting then the previous expression for $N^{*}$, Eq. (13). At a fixed magnitude of $\sigma$, keeping the denominator will increase the value of $N^{*}$.

It should be stressed that, rigorously speaking, from Eq. (16) does not follow a powerlike behavior of $N^{*}$ against $\sigma$. Therefore, we tried to fit the numerical data of Refs. [2, 10 relative to the factor of radiative rate enhancement and the absorption band width using the following parameterization

$$
\begin{gathered}
\frac{N^{*} \sqrt{N^{*}}}{\ln N^{*}-a}=\frac{b}{\sigma^{2}}, \\
\sigma^{*}=c \frac{J \sigma^{2}}{\sqrt{N^{*}}} .
\end{gathered}
$$

The fits were reached provided $a=0.004, b=0.12$ and $c=148$. Note that the numerical factor in $(17 \mathrm{~b})(c=148)$ does not differ drastically from the theoretical value $48 \sqrt{3} \approx 83$.

Finally, we would like to show that for disorder in the interval $0.025<\sigma<0.08$, used in the numerical simulations [2,10], the first term in Eq. (10), neglected by us when making estimates, is smaller than the second one. Their ratio at $K^{\prime}=0$ and $K=2 \pi / N$ is equal to $\pi^{2}\left(1+40 \sigma^{2}\right) /\left(12 N^{2} \sigma^{2}\right)$. Substituting here $N$ by $N^{*} \approx \sigma^{-4 / 3}$, we indeed get for this ratio a small magnitude $\pi^{2}\left(1+40 \sigma^{2}\right) \sigma^{2 / 3} / 12 \ll 1$ when $\sigma$ ranges within the above mentioned interval. Thus, our assumption is self-consistent.

\section{CONCLUSION}

The motional narrowing effect in one-dimensional Frenkel chains with off-diagonal disorder arising from Gaussian fluctuations in the molecular positions is found to be different 
from that for diagonal disorder and for off-diagonal disorder with uncorrelated randomness in the nearest-neighbor couplings. Such distinction is due to the fact that the fluctuations of the dipolar coupling of a given molecule to the adjacent ones are correlated, even if the fluctuations of the molecular positions are completely uncorrelated. Thus, this type of disorder cannot be modeled by uncorrelated randomness in the nearest-neighbor interactions. The estimates of scaling of the optical observables with the degree of disorder based on the new motional narrowing law are found to be in qualitative agreement with those obtained previously in numerical simulations.

\section{ACKNOWLEDGMENTS}

The authors thank A. Sánchez for critical reading of the manuscript. This work was supported by CAM under Project 07N/0034/98. V. A. M. thanks UCM for the support under Sabáticos Complutense as well as partial support from the Russian Foundation for Basic Research under Project 97-03-09221. 


\section{REFERENCES}

$\dagger \quad$ On leave from All-Russian Research Center "Vavilov State Optical Institute", SaintPetersburg, Russia.

[1] E. W. Knapp, Chem. Phys. 85, 73 (1984).

[2] H. Fidder, PhD thesis, Groningen, 1993.

[3] F. C. Spano and J. Knoester, in Advances in Magnetic and Optical Resonance, Vol. 18, ed. W. S. Warren (Academic, New York, 1994), p. 117.

[4] J. Knoester and F. C. Spano, in J aggregates, ed. T. Kobayashi (World Scientific, Singapur, 1996), p. 111.

[5] V. A. Malyshev, Opt. Spektrosk. 84, 235 (1998) [Engl. Transl.: Opt. Spectrosc. 84, 195 (1998)].

[6] M. Wubs and J. Knoester, Chem. Phys. Lett. 284, 63 (1998).

[7] L. D. Bakalis, M. Coca, and J. Knoester, J. Chem. Phys. 110, 2208 (1999).

[8] A. Tilgner, H. P. Trommsdorff, J. M. Zeigler, and R. M. Hochstrasser, J. Lumin. 45, $373(1990)$.

[9] G. G. Kozlov, V. A. Malyshev, F. Domínguez-Adame, and A. Rodríguez, Phys. Rev. B 58, 5367 (1998).

[10] H. Fidder, J. Knoester, and D. A. Wiersma, J. Chem. Phys. 95, 7880 (1991).

[11] V. A. Malyshev, J. Lumin. 55, 225 (1993).

[12] V. A. Malyshev, Opt. Spektrosk. 71, 873 (1991) [Engl. Transl.: Opt. Spectrosc. 71, 505 (1991)].

[13] M. Schreiber and Y. Toyozawa, J. Phys. Soc. Jpn. 51, 1528 (1981); 51, 1537 (1981).

[14] A. Boukahil and D. L. Huber, J. Lumin. 45, 13 (1990). 
[15] J. Köhler, A. M. Jayannavar, and P. Reineker, Z. Phys. B 75, 451 (1989).

[16] V. Malyshev and P. Moreno, Phys. Rev. B 51, 14587 (1995). 